\title{
A MODEL-BASED SOLUTION FOR FAULT DIAGNOSIS OF THRUSTER FAULTS: APPLICATION TO THE RENDEZVOUS PHASE OF THE MARS SAMPLE RETURN MISSION
}

\author{
D. Henry ${ }^{1}$, E. Bornschlegl ${ }^{2}$, X. Olive ${ }^{3}$, and C. Charbonnel ${ }^{3}$ \\ ${ }^{1}$ University Bordeaux 1 - IMS-LAPS \\ Bordeaux, France \\ ${ }^{2}$ European Spatial Agency \\ Noordwijk, The Netherlands \\ ${ }^{3}$ Thales Alenia Space \\ Cannes, France
}

\begin{abstract}
This paper addresses the design of model-based fault diagnosis schemes to detect and isolate faults occurring in the orbiter thrusters of the Mars Sample Return (MSR) mission. The proposed fault diagnosis method is based on a $H(0)$ filter with robust poles assignment to detect quickly any kind of thruster faults and a cross-correlation test to isolate them. Simulation results from the MSR "high-fidelity" nonlinear simulator provided by Thales Alenia Space demonstrate that the proposed method is able to diagnose thruster faults with a detection and isolation delay less than $1.1 \mathrm{~s}$.
\end{abstract}

\section{MOTIVATION}

Future sciences space missions require critical autonomous proximity operations, e.g., rendezvous and docking/capture for the MSR mission. Mission safety is usually guaranteed through various modes of satellite operations, with ground intervention, except for the specific critical phases, for which the onboard robustness and onboard fault tolerance/recovery prevails in the dynamics trajectory conditions.

Satellite health (including outages) monitoring is classically performed through a hierarchical implementation of the fault diagnosis and fault tolerance 
in which several levels of faults containements are defined from local component/ equipment up to global system, i. e., through various equipments (sensors like inertial measurement units, thrusters, etc.) redundancy paths. Common Fault Detection, Isolation, and Recovery (FDIR) implementation uses four hierarchical levels with graduated detection/isolation/reaction to faults (see, for instance, $[1,2]$ where fault detection and isolation (FDI) are performed by cross checks, consistency checks, voting mechanisms, etc.). Fixed thresholds (once validated with all the known delays and uncertainties) are used for rapid recognition of out-of-tolerance conditions but their setting tuned to avoid false alarms and to insure acceptable sensitivity to abnormal deviations. Unfortunately, such classical FDIR hierarchical implementation approach does not solve, sufficiently quickly, abnormal dynamics deviation or transient behavior in faulty situations, e.g., for rendezvous safety corridor during critical proximity operations, thus possibly leading to mission loss. Therefore, advanced model-basd FDI and fault tolerant control techniques are specifically developed to safely conjugate onboard (and online) the necessary robustness/stability of the satellite control and the necessary trajectory dynamics and vehicle operations.

The objective of this research is to develop an advanced model-based FDI scheme, able to diagnose thrusters faults of the MSR orbiter, onboard/online and in time within the critical dynamics and operations constraints of the last terminal translation (last $20 \mathrm{~m}$ ) of the MSR rendezvous/capture phase. As mission scenario undertaken, the chaser stays in the rendezvous/capture corridor, such that it is possible to anticipate the necessary recovery actions to successfully meet the capture phase (see Fig. 1 for an illustration). Three main fault profiles are considered:

(1) locked closed thruster failure;

(2) cyclic forces/torques around the desired force/torque profile with small magnitude; and

(3) monopropellant leakage.

The innovation that is pursued with this study is concerning the fault coverage capability and, more particularly, the ability of the fault diagnosis scheme to detect and isolate small faults which have no significative impact on the spacecraft dynamics and/or the guidance, navigation, and control. For instance, a thruster locked closed is more difficult to diagnose because the thruster is not necessary used at the date of the failure, and because the thrusters, when they are used, achieve small pulses whose effect averaged over the control cycle is small. Such faults are highly nondetectable using the standard industrial onboard FDIR techniques and/or ground analysis. Moreover, the uncertainty on the center of mass due to propellant motions in the tanks makes the detection and isolation more challenging. 

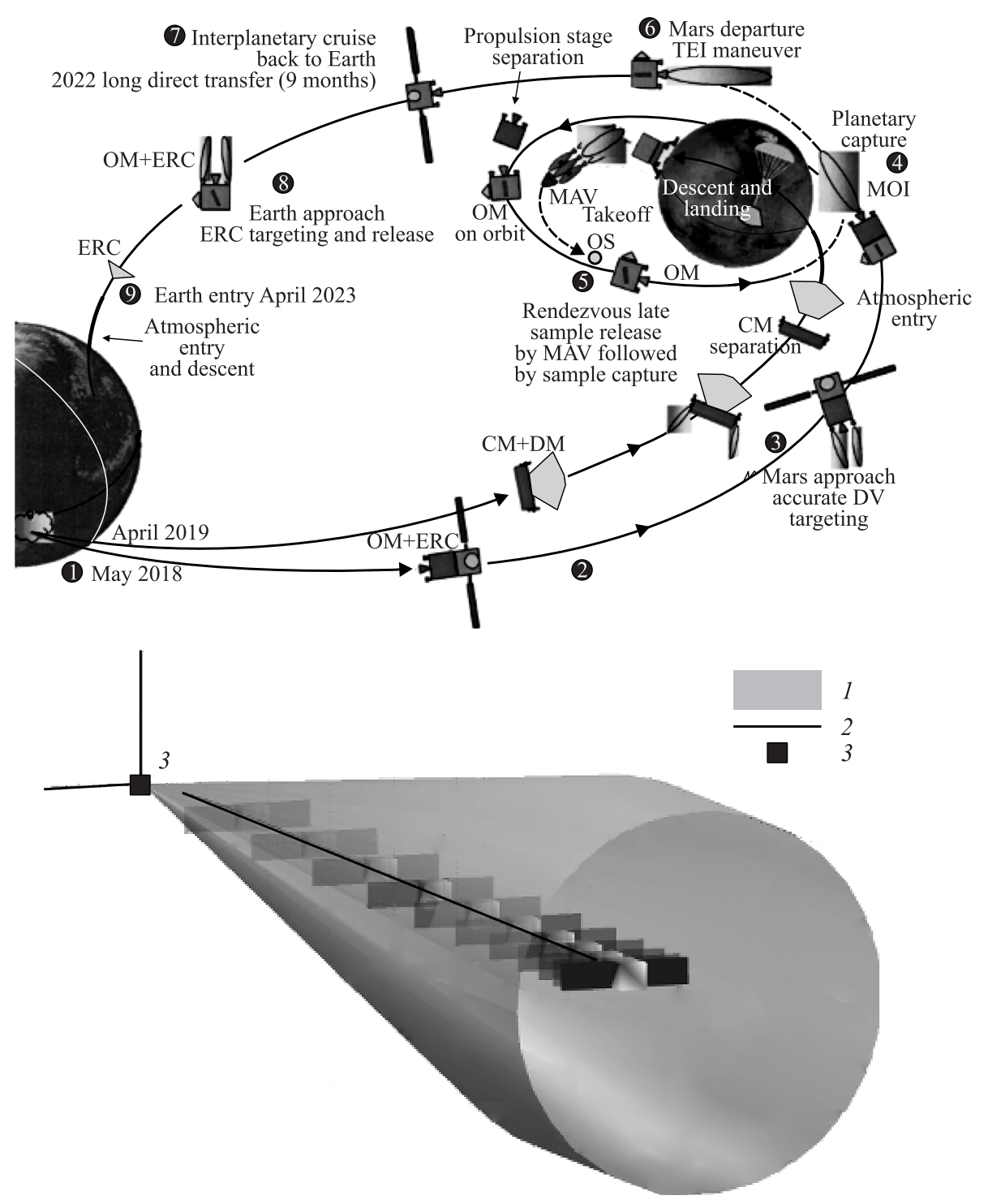

Figure 1 The MSR rendezvous mission: ERC - Earth reentry capsule; OM orbital module; CM - carrier module; DM — descent module; DV - delivery vehicle; MOI - Mars orbit insertion; OS - orbit stabilizer; MAV - Mars ascent vehicle; TEI - trans-Earth injection; 1 - approach coridor; 2 - chaser trajectory; and 3 ascent vehicle 
Numerous fault diagnosis methods are applicable to this problem [3,4]. In fact, most of the model-based diagnostic techniques reported in the literature have the potential to be applied (see [5-8] for good surveys).

In recent years, some effective techniques of the fault detection and diagnosis for satellite attitude control systems based on inertial wheels have been developed (see, for instance, books [9-12] and the references given therein).

The problem of thruster's faults is less considered in the literature. Among the contributions, one can refer to [13] where an iterative learning observer (ILO) is designed to achieve estimation of time-varying thruster faults. The method suggested in $[14,15]$ is based on the so-called unknown input observer technique and is applied to the Mars Express mission. Selected performance criteria are also used, together with Monte Carlo robustness tuning and performance evaluation, to provide fault diagnosis solutions. Henry [16] addressed the problem of thrusters faults in the Microscope satellite and Falcoz et al. [17] considered the problem of faults affecting the micro-Newton colloidal thrust system of the LISA (Laser Interferometer Space Antenna) Pathfinder experiment. Both proposed FDI schemes are based on $H_{\infty} / H_{-}$filters to generate residuals robust against spatial disturbances (i. e., third-body disturbances, $J_{2}$ disturbances, atmospheric drag, and solar radiation pressure), measurement noises, and sensor misalignment phenomena, whilst guaranteeing fault sensitivity performances. Additionally, a Kalman-based projected observer scheme is considered in [17]. $\mathrm{Wu}$ and Saif [18] discuss several fault diagnostic observers using sliding mode and learning approaches.

In this paper, the proposed FDI scheme consists of a $H(0)$ filter with pole assignment which is in charge of residual generation for fault detection. This detection scheme allows to detect quickly any kind of thruster faults. The isolation task is solved using a cross-correlation test between the residual signal and the thrusters. For reduced computational burdens, the isolation test is based on a sliding time window. The key feature of the proposed method is the use of a judiciously chosen linear model for the design of the fault detector, i.e., the model consists of a 6 -order model that takes into account both the rotational and linear translation spacecraft motions. This allows to propose a fault diagnosis solution with reduced computational burdens, which, again, is a prior condition for an onboard implementation. Note that a great advantage of the proposed method is that the use of hyperparameters used to specify the requirements in terms of robustness and fault sensitivity performance allows the proposed technique to be reused for other space missions like ExoMars, Proba3, Mars Express, etc. Furthermore, the existence of formal proofs in terms of fault sensitivity performance (thanks to the $H(0)$ index) allows to pinpoint critical faulty situations. This may lead to a useful tool that can be used to analyze the robustness properties of the GNC against faulty situations prior identified by this tool. Thus, specific Monte Carlo tests can be done before a complete campaign. 
Notations. The Euclidean norm is always used for vectors and is written without a subscript; for example $\|x\|$. Similarly, in the matrix case, the induced vector norm is used: $\|A\|=\bar{\sigma}(A)$ where $\bar{\sigma}(A)$ denotes the maximum singular value of $A$. Signals, for example, $w(t)$ or $w$, are assumed to be of bounded energy, and their norm is denoted by $\|w\|_{2}$, i. e.,

$$
\|w\|_{2}=\left(\int_{-\infty}^{\infty}\|w(t)\|^{2} d t\right)^{1 / 2}<\infty .
$$

Linear models, for example, $P(s)$ or simply $P$, are assumed to be in $\mathbb{R} \mathbb{H}_{\infty}$, real rational functions with

$$
\|P\|_{\infty}=\sup _{\omega} \bar{\sigma}(P(j \omega))<\infty .
$$

In accordance with the induced norm,

$$
\|P\|_{-}=\inf _{\omega \in \Omega} \underline{\sigma}(P(j \omega))
$$

is used to denote the smallest gain of a transfer matrix $P$. Here, $\underline{\sigma}(P(j \omega))$ denotes the minimum nonzero singular value of matrix $P(j \omega)$ and $\Omega=\left[\omega_{1} ; \omega_{2}\right]$ the evaluated frequency range in which $\underline{\sigma}(P(j \omega)) \neq 0$. As a direct extension, the $H(0)$ gain of a multiple-input multiple-output (MIMO) filter is defined according to $\|P\|_{0}=\lim _{\omega \rightarrow 0} \underline{\sigma}(P(j \omega)) \neq 0$ which is known as the zero frequency gain (dcgain). Linear Fractional Representations (LFRs) are extensively used in the paper. For appropriately dimensioned matrices $N$ and

$$
M=\left(\begin{array}{ll}
M_{11} & M_{12} \\
M_{21} & M_{22}
\end{array}\right)
$$

the lower LFR is defined according to

$$
F_{l}(M, N)=M_{11}+M_{12} N\left(I-M_{22} N\right)^{-1} M_{21}
$$

and the upper LFR according to

$$
F_{u}(M, N)=M_{22}+M_{21} N\left(I-M_{11} N\right)^{-1} M_{12},
$$

under the assumption that the involved matrix inverses exist.

\section{MATERIAL BACKGROUNDS}

Consider a dynamical system subject to $q_{f}$ faults $f_{i}(t), i=1, \ldots, q_{f}$. The robust fault detection problem concerns the detection of $f_{i}(t) \neq 0$ while guaranteeing 


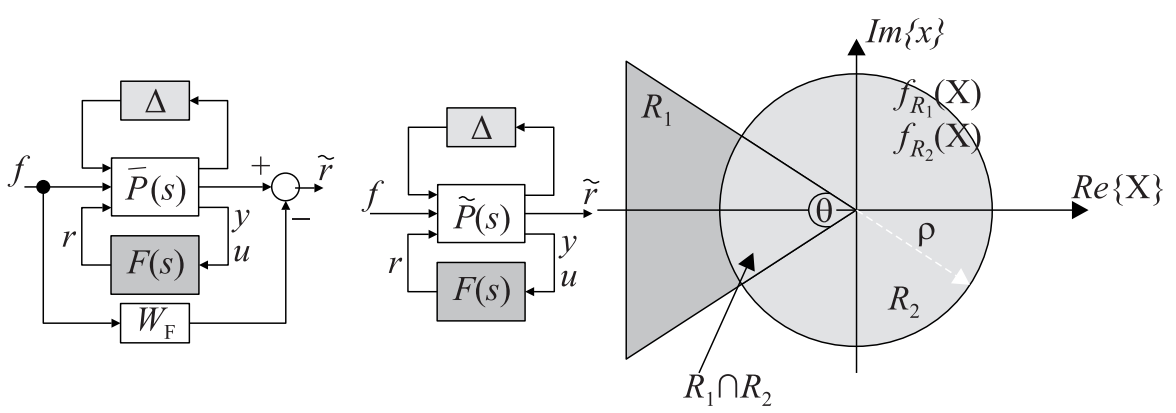

(a)

(b)

Figure 2 The fault detector design problem: $H(0)(a)$ and poles assignment $(b)$ specifications

some robustness performance level to disturbances and model perturbations.

To formulate this problem, consider the uncertain model in the LFR form, i. e., all uncertain parameters and model perturbations have been "pulled out" so that the system's model appears as a nominal model $P$ subject to an artificial feedback $\Delta$ (see Fig. 2 for easy reference):

$$
\left(\begin{array}{c}
y(s) \\
u(s)
\end{array}\right)=F_{u}(P(s), \Delta)\left(\begin{array}{c}
d(s) \\
f(s)
\end{array}\right) .
$$

In this formulation, $x \in \mathbb{R}^{n}, u \in \mathbb{R}^{p}$, and $y \in \mathbb{R}^{m}$ denote the state vector associated to the transfer function $P$ and the input and the output vectors, respectively; $d \in \mathbb{R}^{q_{d}}$ is the vector of all disturbance inputs; $P$ denotes a linear time invariant (LTI) model that includes a control law model (say $u(s)=K(s) y(s)$ where $K$ denotes the controller); and $\Delta$ is the block diagonal operator that behaves to the structure $\underline{\Delta}$ defined as follows:

$$
\begin{array}{r}
\underline{\Delta}=\left\{\text { block diag }\left(\delta_{1}^{r} I_{k_{1}}, \ldots, \delta_{m_{r}}^{r} I_{k_{m_{r}}}, \delta_{1}^{c} I_{k_{m_{r}+1}}, \ldots, \delta_{m_{c}}^{c} I_{k_{m_{r}+m_{c}}}, \Delta_{1}^{C}, \ldots, \Delta_{m_{C}}^{C}\right)\right. \\
\left.\delta_{i}^{r} \in \mathbf{R}, \delta_{i}^{c} \in \mathbb{C}, \Delta_{i}^{C} \in \mathbb{C}\right\}
\end{array}
$$

Here, $\delta_{i}^{r} I_{k_{i}}, i=1, \ldots, m_{r}, \delta_{j}^{c} I_{k_{m_{r}+j}}, j=1, \ldots, m_{c}$, and $\Delta_{l}^{C}, l=1, \ldots, m_{C}$, are known, respectively, as the "repeated real scalar" blocks, the "repeated complex scalar" blocks, and the "full complex" blocks. It is assumed that all model perturbations are represented by $\Delta$ so that $\|\Delta\|_{\infty} \leq 1$. This can be assumed without loss of generality since the model $P$ can always be scaled.

Now, let consider the following general form of a residual vector:

$$
r(s)=F(s)\left(\begin{array}{c}
y(s) \\
u(s)
\end{array}\right), \quad r \in \mathbb{R}^{q_{r}}
$$


The residual generation design problem of interest in can be formulated as follows.

Problem 1. Let the LFR model $F u(P(s), \Delta)$ be robustly stable (this can be done without loss of generality since $P$ may include a controller $K$ ) and the fault $f_{i}$ be observable from the output $y$. These assumptions are prior conditions for the fault detection problem to be well posed. Consider the residual vector $r$ defined by Eq. (2). The aim is to derive the state space matrices $A_{F}, B_{F}, C_{F}$, and $D_{F}$ of the LTI filter $F$ that solve the following optimization problem:

$$
\max _{A_{F}, B_{F}, C_{F}, D_{F}} \varphi \text { s.t. }\left\|T_{f \rightarrow r}\right\|_{0}>\varphi \forall \Delta:\|\Delta\|_{\infty} \leq 1, \lambda_{i}\left(A_{F}\right) \in \mathcal{R} \subseteq \mathcal{D}, \forall i .
$$

Here, $T_{f \rightarrow r}$ denotes the transfer between $f$ and $r$; $\mathcal{D}$ denotes the left-half complex plane; $\lambda_{i}$ refers to the $i$ th eigenvalue of the matric $A_{F}$; and $\varphi$ denotes the fault sensitivity performance index for the residual vector (2). The problem dimensions are $A_{F} \in \mathbb{R}^{n_{F} \times n_{F}}, B_{F} \in \mathbb{R}^{n_{F} \times(m+p)}, C_{F} \in \mathbb{R}^{q_{r} \times n_{F}}$, and $D_{F} \in \mathbb{R}^{q_{r} \times(m+p)}$.

The constraint $\lambda_{i}\left(A_{F}\right) \in \mathcal{R} \subseteq \mathcal{D}, \forall i$, refers to a robust pole assignment constraint and the performance index $\varphi$ guarantees a maximum faults amplification $H(0)$ gain (see the notation paragraph). In other words, the problem is formulated so that the robustness requirements against $d$ are specified through $\mathcal{R}$ while specifying a high fault sensitivity level of the residual vector $r$ through the maximization of $\varphi$. Note that, in practice, $\mathcal{R}$ is a parameter to be selected by the designer since finding an optimal region for $\mathcal{R}$ that guarantees high nuisances rejection is highly related to the system under consideration.

The problem is now to establish a computational procedure for the $H(0)$ and robust pole assignment specifications. Thus, $d$ is ignored from now and this boils down to a new setup as illustrated in Fig. 2 derived from (1) and (2) using some linear algebra manipulations, so that

$$
r(s)=F_{u}\left(F_{l}(\bar{P}(s), F(s)), \Delta\right) f(s) .
$$

\subsection{Semidefinite Programming Formulation of the $H(0)$ FSpecification}

To achieve high fault detection performance, it is proposed in $[4,16,19]$ to introduce a shaping filter $W_{f}$ that allows to specify the fault sensitivity objectives. The solution of Problem 1 is then handled using the following lemma, which is an application of Lemma 2 in [19] to Problem 1 taking into account the definition of the $H(0)$ gain. The proof is omitted here since it can be found in [19].

Lemma 1. Let $W_{f}$ be defined so that $\left\|W_{f}\right\|_{0} \neq 0$. Introduce $W_{F}$, a right invertible transfer matrix so that $\left\|W_{f}\right\|_{0}=(\varphi / \alpha)\left\|W_{F}\right\|_{0}$ and $\left\|W_{F}\right\|_{0}>\alpha$ where 
$\alpha=1+\varphi$. Define the signal $\tilde{r}$ such that $\tilde{r}(s)=r(s)-W_{F}(s) f(s): \tilde{r} \in \mathbb{R}^{q_{r}}$. Then, a sufficient condition for the $H(0)$ specification in (3) to hold is

$$
\left\|T_{f \rightarrow \tilde{r}}\right\|_{\infty}<1, \quad \forall \Delta:\|\Delta\|_{\infty} \leq 1,
$$

where $T_{f \rightarrow \tilde{r}}$ denotes the closed-loop transfer between $\tilde{r}$ and $f$.

Using the above lemma, the filter design problem can be recasted in a fictitious $H_{\infty}$-framework: including $\varphi, \alpha$, and $W_{F}$ into the model $\bar{P}$, one can derive from (4) a new model $\tilde{P}$ so that (see Fig. 2 for easy reference)

$$
\tilde{r}(s)=F_{u}\left(F_{l}(\tilde{P}(s), F(s)), \Delta\right) f(s) .
$$

Noting that $F_{u}\left(F_{l}(\tilde{P}(s), F(s)), \Delta\right)$ is nothing else than the transfer $T_{f \rightarrow \tilde{r}}$, it follows by virtue of Lemma 1 and the small gain theorem that a sufficient condition for the $H(0)$ specification to hold is

$$
\exists F(s):\left\|F_{l}(\tilde{P}(s), F(s))\right\|_{\infty}<1 .
$$

Let $(\tilde{A}, \tilde{B}, \tilde{C}, \tilde{D})$ be the state space matrices of $\tilde{P}$ and consider the following partition of $\tilde{B}, \tilde{C}$, and $\tilde{D}$ :

$$
\tilde{B}=\left(\begin{array}{ll}
\tilde{B}_{1} & \tilde{B}_{2}
\end{array}\right) ; \quad \tilde{C}=\left(\begin{array}{l}
\tilde{C}_{1} \\
\tilde{C}_{2}
\end{array}\right) ; \quad \tilde{D}=\left(\begin{array}{ll}
\tilde{D}_{11} & \tilde{D}_{12} \\
\tilde{D}_{21} & \tilde{D}_{22}
\end{array}\right)
$$

where $\tilde{A} \in \mathbb{R}^{\tilde{n} \times \tilde{n}}, \tilde{D}_{22} \in \mathbb{R}^{(m+p) \times q_{r}}$. It could be verified that $\tilde{B}_{2}=0$ and $\tilde{D}_{22}=0$, showing that the fault detection filter $F$ operates in open-loop vs. the system. Then, using some linear algebra manipulations, it can be verified that the closed-loop model $F_{l}(\tilde{P}(s), F(s))$ admits the state realization $\left(A_{c}, B_{c}, C_{c}, D_{c}\right)$ which is deduced from $\tilde{P}$ and $F$ as follows:

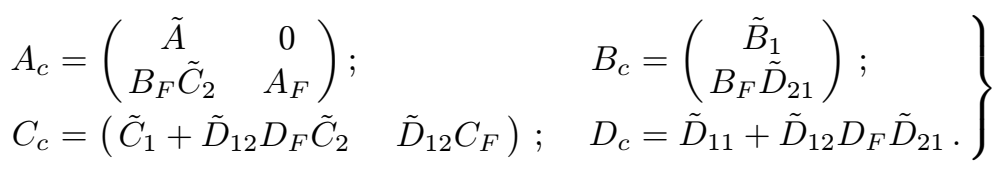

From [20], $F_{l}(\tilde{P}(s), F(s)$ ) is stable (and $F$ is a robustly stable filter due to the triangular structure of $A_{c}$ ) and there exists a solution to (5) if and only if there exists $\gamma<1$ and matrices $\mathbf{A} \in \mathbb{R}^{\tilde{n} \times \tilde{n}}, \mathbf{B} \in \mathbb{R}^{\tilde{n} \times(m+p)}, \mathbf{C} \in \mathbb{R}^{q_{r} \times \tilde{n}}, \mathbf{D} \in \mathbb{R}^{q_{r} \times(m+p)}$, $\mathbf{X}=\mathbf{X}^{\mathrm{T}} \in \mathbb{R}^{\tilde{n} \times \tilde{n}}$, and $\mathbf{Y}=\mathbf{Y}^{\mathrm{T}} \in \mathbb{R}^{\tilde{n} \times \tilde{n}}$ that solves the following semidefinite programming (SDP) problem:

$$
\min \gamma \text { s.t. }
$$

$$
\left(\begin{array}{cccc}
\tilde{A} \mathbf{X}+\mathbf{X} \tilde{A}^{\mathrm{T}} & \mathbf{A}^{\mathrm{T}}+\tilde{A} & \tilde{B}_{1} & \left(\tilde{C}_{1} \mathbf{X}+\tilde{D}_{12} \mathbf{C}\right)^{\mathrm{T}} \\
\mathbf{A}+\tilde{A}^{\mathrm{T}} & \tilde{A}^{\mathrm{T}} \mathbf{Y}+\mathbf{Y} \tilde{A}+\mathbf{B} \tilde{C}_{2}+\left(\mathbf{B} \tilde{C}_{2}\right)^{\mathrm{T}} & \mathbf{Y} \tilde{B}_{1}+\mathbf{B} \tilde{D}_{21} & \left(\tilde{C}_{1}+\tilde{D}_{12} \mathbf{D} \tilde{C}_{2}\right)^{\mathrm{T}} \\
\tilde{B}_{1}^{\mathrm{T}} & \left(\mathbf{Y} \tilde{B}_{1}+\mathbf{B} \tilde{D}_{21}\right)^{\mathrm{T}} & -\gamma I & \left(\tilde{D}_{11}+\tilde{D}_{12} \mathbf{D} \tilde{D}_{21}\right)^{\mathrm{T}} \\
\tilde{C}_{1} \mathbf{X}+\tilde{D}_{12} \mathbf{C} & \tilde{C}_{1}+\tilde{D}_{12} \mathbf{D} \tilde{C}_{2} & \tilde{D}_{11}+\tilde{D}_{12} \mathbf{D} \tilde{D}_{21} & -\gamma I
\end{array}\right)
$$




$$
\left(\begin{array}{cc}
\mathbf{X} & I \\
I & \mathbf{Y}
\end{array}\right)>0
$$

Moreover, $F$ is of full-order, i. e., $n_{F}=\tilde{n}$. The fault detector state space matrices $A_{F}, B_{F}, C_{F}$, and $D_{F}$ are then deduced from $\mathbf{A}, \mathbf{B}, \mathbf{C}, \mathbf{D}, \mathbf{X}$, and $\mathbf{Y}$ according to the following procedure which is a direct application of the procedure proposed in $[20]$ to the considered problem:

(i) find nonsingular matrices $M$ and $N$ to satisfy $M N^{\mathrm{T}}=I-\mathbf{X Y}$ (this can be done easily using the singular value decomposition technique); and

(ii) define the fault detector by

$$
\left.\begin{array}{ll}
D_{F}=\mathbf{D} ; & C_{F}=\left(\mathbf{C}-\mathbf{D} \tilde{C}_{2} \mathbf{X}\right) M^{-\mathrm{T}} ; \\
B_{F}=N^{-1} \mathbf{B} ; & A_{F}=N^{-1}\left(\mathbf{A}-N B_{F} \tilde{C}_{2} \mathbf{X}-\mathbf{Y} \tilde{A} \mathbf{X} M^{-\mathrm{T}}\right) .
\end{array}\right\}
$$

\subsection{Linear Matrix Inequalities Formulation of the Robust Poles Assignment Specification}

Consider now the specification $\lambda_{i}\left(A_{F}\right) \in \mathcal{R} \subseteq \mathcal{D}, \forall i$. Assume that the region $\mathcal{R}$ is formed by the intersection of $N$ elementary linear matrix inequalities (LMI) regions $\mathcal{R}_{i}$, i.e., $\mathcal{R}=\mathcal{R}_{1} \cap \cdots \cap \mathcal{R}_{N}$ (see Fig. 2 for easy reference). Each LMI region $\mathcal{R}_{i}$ is characterized as follows:

$$
\mathcal{R}_{i}=\left\{\chi \in \mathbb{C}: L_{i}+\chi Q_{i}+\chi^{*} Q_{i}^{\mathrm{T}}<0\right\}
$$

where $L_{i}$ and $Q_{i}$ are the real symmetric matrices. The matrix-valued function $f_{\mathcal{R}_{i}}(\chi)=L_{i}+\chi Q_{i}+\chi^{*} Q_{i}^{\mathrm{T}}$ is called the characteristic function of the $i$ th LMI region $\mathcal{R}_{i}$. Then, in [21], it is shown that a sufficient condition for all eigenvalues of $A_{c}$ given by (6), lying in the region $\mathcal{R}$ for all $\Delta \in \Delta:\|\Delta\|_{\infty} \leq 1$, is the existence, for each region $\mathcal{R}_{i}$, of a matrix $P_{i}$ and $\beta<1$ so that

$$
\left(\begin{array}{ccc}
\mathbb{Q}\left(A_{c}, P_{i}\right) & Q_{1 i}^{\mathrm{T}} \otimes\left(P_{i} B_{c}\right) & Q_{2 i}^{\mathrm{T}} \otimes C_{c}^{\mathrm{T}} \\
Q_{1 i} \otimes\left(B_{c}^{\mathrm{T}} P_{i}\right) & -\beta I & I \otimes D_{c}^{\mathrm{T}} \\
Q_{2 i} \otimes C_{c} & I \otimes D_{c} & -\beta I
\end{array}\right)<0, \quad P_{i}>0, \quad i=1, \ldots, N,
$$

where $\otimes$ denotes the Kronecker product of matrices. The matrix $\mathcal{Q}_{\mathcal{R}_{i}}\left(A_{c}, P_{i}\right)$ is defined according to

$$
\mathbb{Q}\left(A_{c}, P_{i}\right)=L_{i} \otimes P_{i}+Q_{i} \otimes\left(P_{i} A_{c}\right)+Q_{i}^{\mathrm{T}} \otimes\left(A_{c}^{\mathrm{T}} P_{i}\right),
$$

$Q_{1 i}^{\mathrm{T}} Q_{2 i}=Q_{i}$ is the factorization of $Q_{i}$ so that $Q_{1 i}$ and $Q_{2 i}$ have full column rank. 
Due to the triangular structure of $A_{c}$, it is obvious that the set of the eigenvalues of $A_{c}$ are equal to the set of the eigenvalues of $\tilde{A}$ and $A_{F}$. Thus, a sufficient condition for all fault detection filter poles lying in the LMI region $\mathcal{R}$ for all $\Delta \in \underline{\Delta}:\|\Delta\|_{\infty} \leq 1$ (i. e., for the robust pole assignment specification to hold) is the existence of a solution to the inequalities (10). Unfortunately, since each inequality constraint involves products of a matrix $P_{i}, i=1, \ldots, N$, and the fault filter variables $A_{F}, B_{F}, C_{F}$, and $D_{F}$, the resulting optimization problem is nonlinear. To reduce the problem to a linear optimization problem, the linearizing change of variables given by (9) can be used.

Let $\tilde{B}_{1}, \tilde{C}_{1}, \tilde{D}_{11}, \tilde{D}_{12}$, and $\tilde{D}_{21}$ be partitioned according to the dimension of $\Delta$ such that

$$
\begin{aligned}
& \tilde{B}_{1}=\left(B_{\Delta} B_{f}\right) ; \\
& \tilde{C}_{1}=\left(\begin{array}{c}
C_{\Delta} \\
C_{r}
\end{array}\right) ; \\
& \tilde{D}_{11}=\left(\begin{array}{cc}
D_{\Delta \Delta} & D_{\Delta f} \\
D_{r \Delta} & D_{r f}
\end{array}\right) ; \\
& \tilde{D}_{12}=\left(\begin{array}{c}
D_{1 \Delta} \\
D_{1 r}
\end{array}\right) ; \\
& \tilde{D}_{21}=\left(D_{2 \Delta} D_{2 f}\right) \text {. }
\end{aligned}
$$

It follows that all eigenvalues of $A_{F}$ lye in the region $\mathcal{R}$ for all $\Delta \in \underline{\Delta}:\|\Delta\|_{\infty} \leq 1$ if there exist $\beta<1, \mathbf{A}, \mathbf{B}, \mathbf{C}, \mathbf{D}$, and $\mathbf{X}_{i}=\mathbf{X}_{i}^{\mathrm{T}} \in \mathbb{R}^{\tilde{n} \times \tilde{n}}, \mathbf{Y}_{i}=\mathbf{Y}_{i}^{\mathrm{T}} \in \mathbb{R}^{\tilde{n} \times \tilde{n}}$, $i=1, \ldots, N$, that solve the following SDP problem:

$$
\min \beta \text { s.t. }\left(\begin{array}{ccc}
L_{i} \otimes \Psi\left(\mathbf{X}_{i}, \mathbf{Y}_{i}\right)+Q_{i} \otimes \Phi_{A}+Q_{i}^{\mathrm{T}} \otimes \Phi_{A}^{\mathrm{T}} & Q_{1 i}^{\mathrm{T}} \otimes \Phi_{B} & Q_{2 i}^{\mathrm{T}} \otimes \Phi_{C}^{\mathrm{T}} \\
Q_{1 i} \otimes \Phi_{B}^{\mathrm{T}} & -\beta I & I \otimes \Phi_{D}^{\mathrm{T}} \\
Q_{2 i} \otimes \Phi_{C} & I \otimes \Phi_{D} & -\beta I
\end{array}\right)
$$

with

$$
\begin{aligned}
\Psi\left(\mathbf{X}_{i}, \mathbf{Y}_{i}\right) & =\left(\begin{array}{cc}
\mathbf{X}_{i} & I \\
I & \mathbf{Y}_{i}
\end{array}\right)>0 \\
\Phi_{A} & =\left(\begin{array}{cc}
\tilde{A} \mathbf{X}_{i} & \tilde{A} \\
\mathbf{A} & \mathbf{Y}_{i} \tilde{A}+\mathbf{B} \tilde{C}_{2}
\end{array}\right) ; \\
\Phi_{B} & =\left(\begin{array}{c}
B_{\Delta} \\
\mathbf{Y}_{i} B_{\Delta}+\mathbf{B} D_{2 \Delta}
\end{array}\right) ; \\
\Phi_{C} & =\left(\begin{array}{c}
\left.C_{\Delta} \mathbf{X}_{i}+D_{1 \Delta} \mathbf{C} C_{\Delta}+D_{1 \Delta} \mathbf{D} \tilde{C}_{2}\right) \\
\Phi_{D}
\end{array}=D_{\Delta \Delta}+D_{1 \Delta} \mathbf{D} D_{2 \Delta}, i=1, \ldots, N\right.
\end{aligned}
$$


Remark 1. From the above developments, problem 1 can be solved by jointly solving the SDP problems (7)-(8) and (11). This boils down to a multiobjective optimization problem in the form

$$
\min \varepsilon \gamma+(1+\varepsilon) \beta \quad \text { s.t. (7)-(8) and (11), }
$$

whereby the choice of $\varepsilon$ is guided by the Pareto optimal points. However, in practice, $\beta$ is better considered as a parameter to be fixed to $\beta=1$. Thus, the resulting optimization problem looks for the best achievable $H(0)$ objective whereas the robust pole assignment constraint is enforced. Any $\gamma<1$ indicates that the obtained solution is admissible for problem 1 . However, $\gamma \approx 1^{-}$is required in order to obtain a low conservative solution. Furthermore, as it is now well known, all aforementioned inequalities must be solved by using a single Lyapunov matrix for feasibility reasons. This boils down to the additional constraints $\mathbf{X}_{1}=\cdots=\mathbf{X}_{N}=\mathbf{X}$ and $\mathbf{Y}_{1}=\cdots=\mathbf{Y}_{N}=\mathbf{Y}$.

\section{APPLICATION TO THE MARS SAMPLE RETURN MISSION}

\subsection{Modeling the Orbiter Dynamics During the Rendezvous Phase}

In the interest of brevity, throughout this section, an earnest attempt will be made to avoid duplicating material presented in the extensive aerospace literature about modeling the satellite's dynamics (see, for example, [9, 22-25]).

The motion of the orbiter is derived from the second Newton law. To proceed, let $a, m, \mathcal{G}$, and $m_{M}$ denote the orbit of the target, the mass of the orbiter, the gravitational constant, and the mass of the planet Mars. Then, the orbit of the rendezvous being circular, the velocity of any object (e.g., the chaser and the target) is given be the relation $\sqrt{\mu / a}$ where $\mu=\mathcal{G} m_{M}$. Let $\mathcal{R}_{l}:\left(O_{t g t}, \overrightarrow{X_{l}}, \overrightarrow{Y_{l}}, \overrightarrow{Z_{l}}\right)$ be the frame attached to the target and oriented as shown in Fig. 3. Because the linear velocity of the target is given by the relation $a \dot{\theta}$ in the inertial frame $\mathcal{R}_{i}:\left(O_{M}, \overrightarrow{X_{i}}, \overrightarrow{Y_{i}}, \vec{Y}_{i}\right)$ (those that are attached to the center of Mars, see Fig. 3), it follows:

$$
a \dot{\theta}=\sqrt{\frac{\mu}{a}} \Rightarrow n=\sqrt{\frac{\mu}{a^{3}}} .
$$

During the rendezvous phase, it is assumed that the orbiter motion is due to the following four forces:

(1) the Mars attraction force $\overrightarrow{F_{a}}$ given in $\mathcal{R}_{l}$ by

$$
\overrightarrow{F_{a}}=-m \frac{\mu}{\left((a+\xi)^{2}+\eta^{2}+\zeta^{2}\right)^{3 / 2}}\left((a+\xi) \overrightarrow{X_{l}}+\eta \overrightarrow{Y_{l}}+\zeta \overrightarrow{Z_{l}}\right)
$$




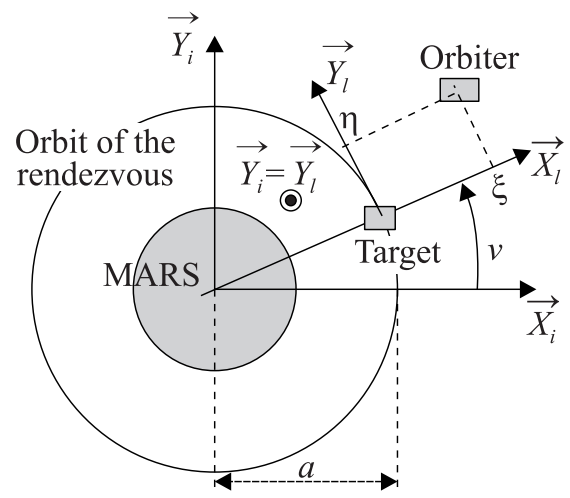

Figure 3 The rendezvous orbit and associated frames

where $\xi, \eta$, and $\zeta$ denote the three-dimensional (3D) position of the orbiter (assumed to be a ponctual mass) in $\mathcal{R}_{l}$;

(2) the inertial force

$$
\overrightarrow{F_{e}}=m\left(n^{2} \xi \overrightarrow{X_{l}}+n^{2} \eta \overrightarrow{Y_{l}}+0 \overrightarrow{Z_{l}}\right)
$$

(3) the Coriolis force $\vec{F}_{c}$ that is given in $\mathcal{R}_{l}$ by

$$
\overrightarrow{F_{c}}=m\left(2 n \dot{\eta} \overrightarrow{X_{l}}-2 n \dot{\xi} \overrightarrow{Y_{l}}+0 \vec{Z}_{l}\right)
$$

(4) the forces due to the thrusters

$$
\overrightarrow{F_{\mathrm{thr}}}=F_{\xi} \overrightarrow{X_{l}}+F_{\eta} \overrightarrow{Y_{l}}+F_{\zeta} \overrightarrow{Z_{l}}
$$

Then, from the second Newton law, it follows:

$$
\left.\begin{array}{l}
\ddot{\xi}=n^{2} \xi+2 n \dot{\eta}-\frac{\mu}{\left((a+\xi)^{2}+\eta^{2}+\zeta^{2}\right)^{3 / 2}}(a+\xi)+\frac{F_{\xi}}{m} ; \\
\ddot{\eta}=n^{2} \eta-2 n \dot{\xi}-\frac{\mu}{\left((a+\xi)^{2}+\eta^{2}+\zeta^{2}\right)^{3 / 2}} \eta+\frac{F_{\eta}}{m} ; \\
\ddot{\zeta}=-\frac{\mu}{\left((a+\xi)^{2}+\eta^{2}+\zeta^{2}\right)^{3 / 2}} \zeta+\frac{F_{\zeta}}{m} .
\end{array}\right\}
$$

Because the distance between the target and the orbiter is smaller than the orbit $a$, it is possible to derive the so-called Hill-Clohessy-Wiltshire equations from Eqs. (12) by means of a first-order approximation. This boils down to a linear six-order state space model whose input vector is $u=\left(F_{\xi} F_{\eta} F_{\zeta}\right)^{\mathrm{T}}$ and 
state vector $x=(\xi \eta \zeta \dot{\xi} \dot{\eta} \dot{\zeta})^{\mathrm{T}}$. Now, projecting the thrust forces due to the eight thrusters that equip the orbiter into the frame $\mathcal{R}_{l}$, it follows from (12):

$$
\left.\begin{array}{l}
\dot{x}=A x+B R\left(\hat{Q}_{\mathrm{tgt}}(t), \hat{Q}_{\mathrm{chs}}(t)\right) M u_{\mathrm{thr}}(t)+B_{w} w(t) ; \\
y=C x+n .
\end{array}\right\}
$$

Here, $\hat{Q}_{\text {tgt }} \in \mathbb{R}^{4}$ and $\hat{Q}_{\text {chs }} \in \mathbb{R}^{4}$ denote the attitude's quaternion of the target and the orbiter, respectively. These quaternions are denoted as estimates since they are provided (i. e., estimated) by the navigation module. In (13), $M \in \mathbb{R}^{3 \times 8}$ refers to the (static) allocation module, $u_{\mathrm{thr}} \in \mathbb{R}^{8}$ refers to the thrusters input, and $R(\cdot)$ is used for a rotation matrix; $x \in \mathbb{R}^{6}$ is the state vector defined previously, $y \in \mathbb{R}^{3}$ refers to the $3 \mathrm{D}$ positions measured by means of a light detecting and ranging (LIDAR) unit, and $w \in \mathbb{R}^{3}$ refers to the spatial disturbances. The considered disturbances in this study are solar radiations, gravity gradient, and atmospheric drag. In (13), $n$ denotes the measurement noise assumed to be a white noise with very small variance due to the technology used for the design of the LIDAR; $A, B$, and $C$ are the matrices of adequate dimension. With regard to the faults, any kind of faults occurring in the thrusters is interesting. Such faults can be modeled in a multiplicative manner according to (the index " $f$ " is used to outline the faulty case):

$$
\left.\begin{array}{c}
u_{\mathrm{thr}}^{f}(t)=\left(I_{8}-\Psi(t)\right) u_{\mathrm{thr}}(t) ; \\
\Psi(t)=\operatorname{diag}\left\{\psi_{i}(t)\right\}: 0 \leq \psi_{i}(t) \leq 1, \quad i=1, \ldots, 8,
\end{array}\right\}
$$

where $\psi_{i}, i=1, \ldots, 8$ are unknown. In $(14), \Psi(t)$ models thruster faults, e.g., a locked-in-placed fault can be modeled by $\Psi_{i}(t)=1-c / u_{\mathrm{ths}}(t)$ where $c$ denotes a constant value (the particular values $c=\{0,1\}$ allows to consider open/closed faults) whereas a fix value of $\Psi_{i}$ models a loss of efficiency of the $i$ th thruster; $\Psi(t)=0 \forall t$ means that no fault occurs in the thrusters.

Then, taking into account some unknown but bounded delays induced by the electronic devices and the uncertainties on the thruster rise times due to the thruster modulator unit that is modeled here to be a constant gain with unknown but bounded time delay

$$
\tau=\tau_{0} \pm \delta_{\tau}:\left|\delta_{\tau}\right| \leq \bar{\delta}_{\tau},
$$

the motion of the orbiter during the rendezvous can be modeled in both fault free (i. e., $\Psi=0$ ) and faulty (i. e., $\Psi \neq 0$ ) situations according to:

$$
\left.\begin{array}{l}
\dot{x}=A x+B R\left(\hat{Q}_{\mathrm{tgt}}(t), \hat{Q}_{\mathrm{chs}}(t)\right) M(I-\Psi(t)) u_{\mathrm{thr}}(t-\tau)+B_{w} w(t) ; \\
y=C x+n
\end{array}\right\}
$$


where

$$
\tau=\tau_{0} \pm \delta_{\tau}:\left|\delta_{\tau}\right| \leq \overline{\delta_{\tau}} ; \quad \Psi(t)=\operatorname{diag}\left(\psi_{i}(t)\right): 0 \leq \psi_{i}(t) \leq 1, \quad i=1, \ldots, 8
$$

Now, using a Pade approximation of the time delay $\tau$, considering $R\left(\hat{Q}_{\mathrm{tgt}}(t), \hat{Q}_{\mathrm{chs}}(t)\right) M u_{\mathrm{thr}}(t)$ as the input vector $u(t)$ and approximating the fault model $R\left(\hat{Q}_{\mathrm{tgt}}(t), \hat{Q}_{\mathrm{chs}}(t)\right) M \Psi(t) u_{\mathrm{thr}}(t)$ in terms of additive faults $f(t) \in \mathbb{R}^{3}$ acting on the state via a constant distribution matrix $K_{f}$ (then $K_{f}=B$ ), it follows that the overall model of the orbiter dynamics that takes into account both the attitude $\left(Q_{\text {chs }}(t)\right)$ and the relative position $(x(t))$ of the orbiter can be written in the form (1) with $d=n$. The uncertain parameter $\tau$ has been "pulled out" so that system (15) appears as the nominal model $P$ subject to an artificial feedback $\Delta=\delta_{\tau} I_{8}$, that is,

$$
y(s)=F_{u}(P(s), \Delta)\left(\begin{array}{l}
f(s) \\
u(s)
\end{array}\right)+n(s)
$$

where

$$
u(s)=K(s) y(s) ; \quad \Delta=\delta_{\tau} I_{8}:\|\Delta\| \leq 1
$$

\subsection{Design of the Fault Detection and Isolation Scheme}

\subsubsection{Design of the fault detection filter}

The robust fault detection scheme presented in section 2 is now considered. The problem dimensions are $q_{f}=3, q_{r}=3, m=3, p=3$. The shaping filter $W_{f}$ involved in lemma 1 is chosen to be a low pass filter of the first order with $H(0)$ gain the highest possible. With regards to the robust pole clustering constraint, it is required robust pole clustering in the LMI region defined as the intersection of the two following regions, i. e., $\mathcal{R}=\mathcal{R}_{1} \cap \mathcal{R}_{2}$ :

(1) $\mathcal{R}_{1}$ : disk with center $(-q, 0)$ and radius $\rho$ (to prevent fast dynamics). This region is defined according to

$$
\mathcal{R}_{1}=\left\{\chi \in \mathbb{C}:\left(\begin{array}{cc}
-\rho & q \\
q & -\rho
\end{array}\right)+\chi\left(\begin{array}{ll}
0 & 1 \\
0 & 0
\end{array}\right)+\chi^{*}\left(\begin{array}{ll}
0 & 0 \\
1 & 0
\end{array}\right)<0\right\}
$$

where $q=0.5$ and $\rho=1$. By this choice, it is required all eigenvalues of $A_{F}$ to be close to -0.5 ; and 
(2) $\mathcal{R}_{2}$ : shifted conic sector with apex at $\omega$ and angle $\theta$. Region $\mathcal{R}_{2}$ is characterized according to

$$
\begin{aligned}
& \mathcal{R}_{2}=\left\{\chi \in \mathbb{C}:\left(\begin{array}{cc}
-2 \omega \cos (\theta) & 0 \\
0 & -2 \omega \cos (\theta)
\end{array}\right)+\chi\left(\begin{array}{cc}
\cos (\theta) & \sin (\theta) \\
-\sin (\theta) & \cos (\theta)
\end{array}\right)\right. \\
& \left.+\chi^{*}\left(\begin{array}{cc}
\cos (\theta) & -\sin (\theta) \\
\sin (\theta) & \cos (\theta)
\end{array}\right)<0\right\}
\end{aligned}
$$

where the numerical values of $\omega$ and $\theta$ are fixed, respectively, to $\omega=10$ and $\theta=5^{\circ}$. This particular region is chosen to maintain a suitable damping ratio. Note that as (7)-(8) enforce filter stability, it is inconsequential that the LMI region $\mathcal{R}$ intersects the right half-plane.

Following the discussion in section 2, the fault detection filter state-space matrices $A_{F}, B_{F}, C_{F}$, and $D_{F}$ are computed so that inequalities (7)-(8) and (11) are satisfied. As expected, the poles of the so-computed filter are found to be close to -0.5 . Figure 4 illustrates the principal gains $T_{u \rightarrow r}(j \omega)$ (the transfer between the inputs $u$ and the residuals $r$ ) and $T_{y \rightarrow r}(j \omega)$ (the transfer between the measurements $y$ and the residuals $r$ ) of the computed filter $F$. As it can be seen, $T_{u \rightarrow r}(j \omega)$ behaves like a low pass filter, whereas $T_{y \rightarrow r}(j \omega)$ behaves like a high pass filter. Furthermore, it can be noted that the gains of $T_{y \rightarrow r}(j \omega)$ are always lower than 1 showing that the measurement noise is not amplified on the residuals $r(t)$ (Figs. 5-9).

\subsubsection{The isolation strategy}

With regards to the fault isolation task and based on the method proposed in [16], the following normalized cross-correlation criterion between the residuals $r$ and the associated controlled thrusters open rate $u_{\mathrm{thr}_{i}}$ is used here:

$$
\begin{aligned}
& i(k)=\operatorname{argmin} \frac{1}{N} \sum_{k=\tau-N}^{\tau}\left(r_{j}(k)-\bar{r}\right)\left(u_{\mathrm{thr}_{i}}(k)-\bar{u}_{\mathrm{thr}_{i}}\right), \\
& i=1, \ldots, 8, \quad j \in\{1,2,3\}, \quad t=k T_{s} .
\end{aligned}
$$

Here, $\bar{r}, \overline{u_{\mathrm{thr}_{i}}}, i=1, \ldots, 8$, and $T_{s}$ denote the mean values of $r$ and $u_{\mathrm{thr}_{i}}$, $i=1, \ldots, 8$, and the navigation module sampling period. For real-time reason, this criterion is computed on a $N$-length sliding window. The resulting index $i(k)$ also refers to the identified faulty thruster. A key feature of this isolation strategy is that it is static and, then, has low computational burdens. 


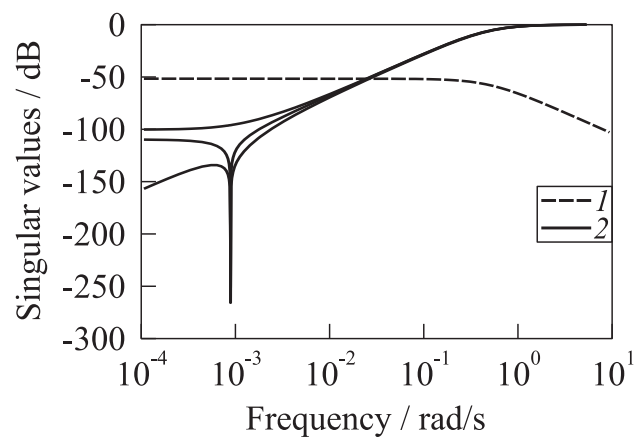

Figure 4 Principal gains of the filter $F: 1-T_{u \rightarrow r}$; and $2-T_{y \rightarrow r}$ for some faulty situations
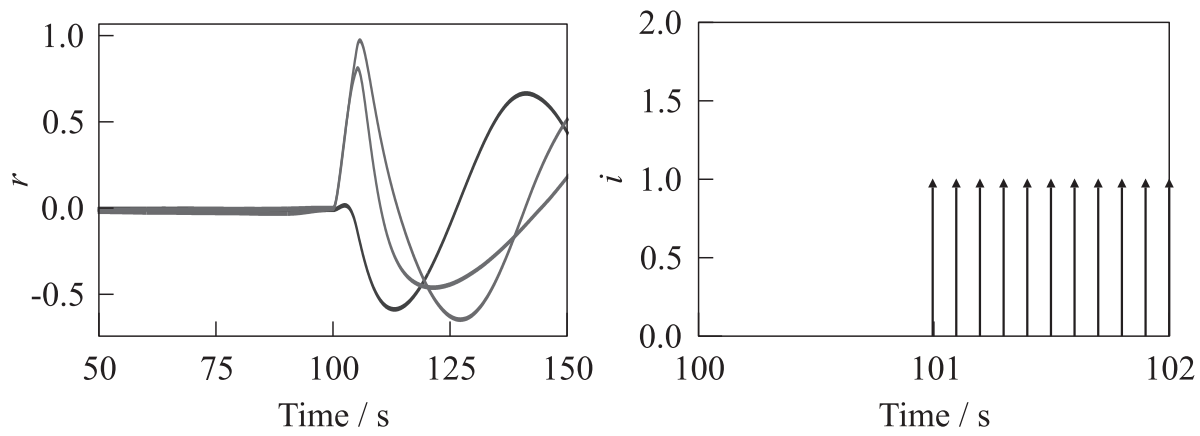

Figure 5 Behavior of $r(t)$ and $i(t)$ for fault in thruster No. 1
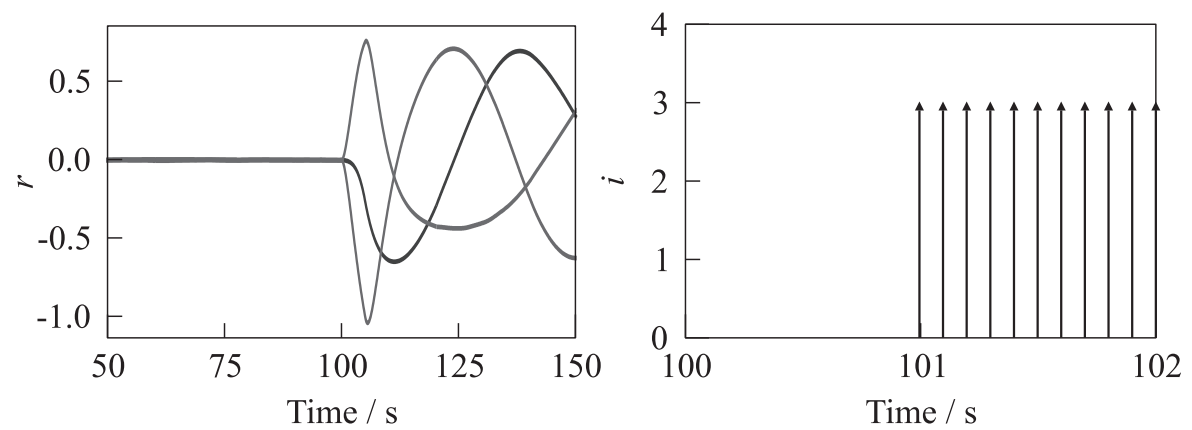

Figure 6 Behavior of $r(t)$ and $i(t)$ for fault in thruster No. 3 

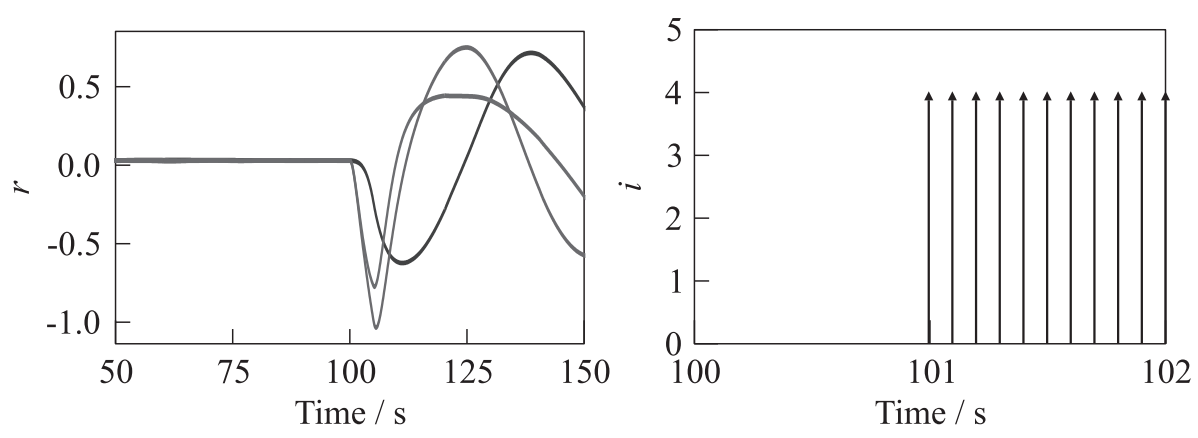

Figure 7 Behavior of $r(t)$ and $i(t)$ for fault in thruster No. 4
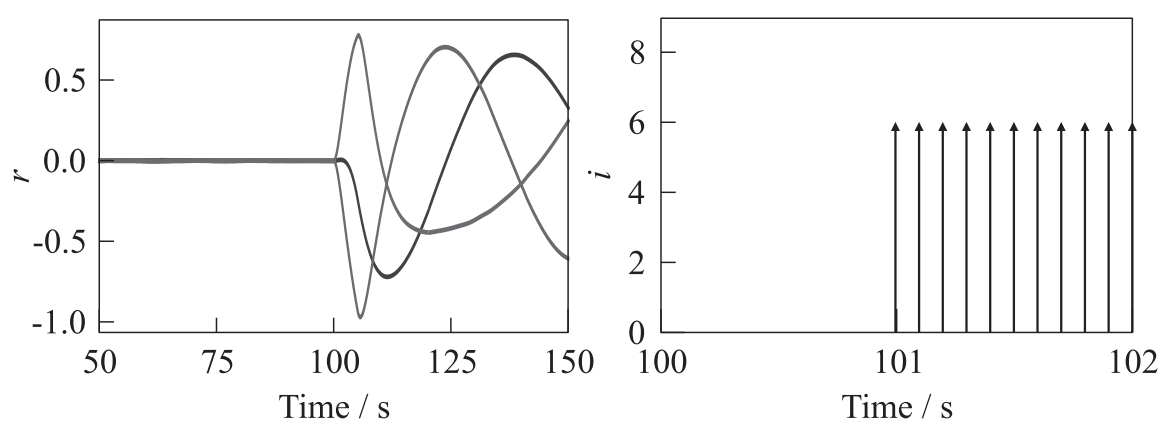

Figure 8 Behavior of $r(t)$ and $i(t)$ for fault in thruster No. 6
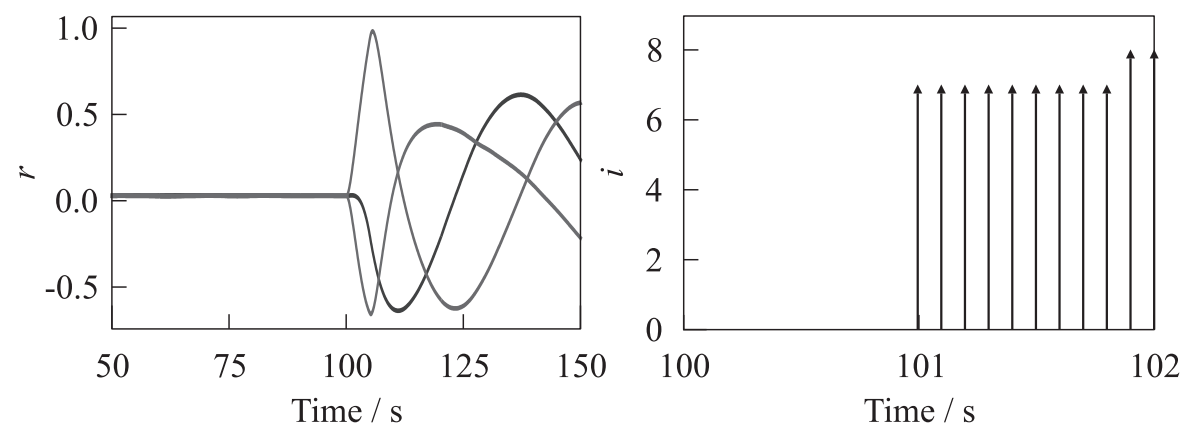

Figure 9 Behavior of $r(t)$ and $i(t)$ for fault in thruster No. 7 


\subsection{Simulation Results}

The fault detection filter $F$ is converted to discrete-time using a Tustin approximation and implemented within the nonlinear simulator of the MSR mission. The simulated faults correspond to a single thruster opening at $100 \%$ during the last $20 \mathrm{~m}$ of the rendezvous. To make a final decision about the fault, a sequential Wald decision test applied to $\|r(t)\|_{2}$ is implemented within the simulator. The probabilities of nondetection and false alarms have been fixed to $0.1 \%$. Also, the isolation strategy is implemented within the nonlinear simulator with $j=1$ (see Eq. (16)).

Figures 5-9 illustrate the behavior of the residual $r(t)$ and the isolation criteria $i(t)$ for some faulty situations. For each simulation, the fault occurs at $t=100 \mathrm{~s}$ and is maintained. The strategy works as follows: as soon as the fault is declared by the decision test, the cross-correlation criterion (16) is computed. As it can be seen in the figures, all thruster faults are successfully detected and isolated by the FDI unit with a detection and isolation delay less than $1.1 \mathrm{~s}$. Note that such a strategy succeeds since both the rotational $\left(Q_{\mathrm{chs}}(t)\right)$ and linear translation $(x(t))$ orbiter motions have been considered. By this way, the effects that faults have on both the orbiter attitude and translation motion are taken into account.

\section{CONCLUDING REMARKS}

This paper addressed the design of robust model-based fault diagnosis schemes to detect and isolate faults occurring in the orbiter's thrusters unit of the MSR mission. The presented study focused on the orbiter spacecraft during the rendezvous phase with the Mars ascent vehicle. The proposed fault diagnosis scheme consists of a $H(0)$ filter with robust poles assignment which is in charge of residual generation for fault detection. The isolation task is solved using a crosscorrelation test between the residuals and the thrusters signals. For reduced computational burdens, the isolation test is based on a sliding time window. The key feature of the proposed method is the use of a judiciously chosen linear model for the design of the filter, i. e., the model consists of a 6-order model given in a judiciously chosen frame that takes into account both the rotational and linear translation spacecraft motions. This allows to propose a fault diagnosis solution with reduced computational burdens that is then thought to be a potential candidate for onboard implementation.

\section{REFERENCES}

1. Bornschleg, E. 2008. FDIR requirements and rational - ESA R\&D activities overview for GNC and software. Toulouse, France: CCT CNES. 
2. Olive, X. 2010. FDI(R) for satellite at Thales Alenia Space: How to deal with high availability and robustness in space domain? Conference on Control and FaultTolerant Systems (SysTol'10) Proceedings. Nice, France: IEEE. 837-42.

3. Henry, D., S. Simani, and R. Patton. 2010. Fault detection and diagnosis for aeronautic and aerospace missions in fault tolerant flight control: A benchmark challenge. Springer Verlag.

4. Henry, D. 2011. A norm-based point of view for fault diagnosis: Application to aerospace missions. Automatic Control Aerospace 4(1). (Online Journal.)

5. Venkatasubramanian, V., R. Rengaswamy, K. Yin, and S. Kavuri. 2003. A review of process fault detection and diagnosis. Part 1: Quantitative model-based methods. Comput. Chem. Eng. 27(3):293-311.

6. Blanke, M., M. Kinnaert, M. Lunze, and M. Staroswiecki. 2003. Diagnosis and fault tolerant control. New York: Springer.

7. Isermann, R. 2005. Model-based fault detection and diagnosis - status and applications. Ann. Rev. Control 29:71-85.

8. Ding, S. 2008. Model-based fault diagnosis techniques — design schemes, algorithms and tools. New York: Springer.

9. Wertz, J. R., and W. J. Larson. 1999. Space mission analysis and design. 3rd ed. New York: Springer-Verlag, LLC.

10. Hao, H., Z. Sun, and Y. Zhang. 2004. Fault diagnosis on satellite attitude control with dynamic neural network. Lecture notes in computer science ser. Berlin/Heidelberg: Springer.

11. Azarnoush, H. 2010. Fault diagnosis in spacecraft attitude control system: A modelbased approach. LAP LAMBERT Academic Publishing.

12. Mirzaee, A., and A. Foruzantabar. 2011. Nonlinear-fuzzy based design actuator fault diagnosis for the satellite attitude control system. IFIP Advances in Information and Communication Technology. Boston: Springer.

13. Chen, W., and M. Saif. 2007. Observer-based fault diagnosis of satellite systems subject to time-varying thruster faults. J. Dyn. Syst. Measurement Control. Tr. ASME 129(3):352-56.

14. Patton, R., F. Uppal, S. Simani, and B. Polle. 2008. Reliable fault diagnosis scheme for a spacecraft attitude control system. Proc. IMechE. Part 0: J. Risk Reliability 222:139-52.

15. Patton, R., F. Uppal, S. Simani, and B. Polle. 2010. Robust FDI applied to thruster faults of a satellite system. Control Eng. Practice 18(9):1093-109.

16. Henry, D. 2008. Fault diagnosis of the microscope satellite actuators using $H_{\infty} / H_{-}$ filters. AIAA J. Guidance Control Dyn. 31(3):699-711.

17. Falcoz, A., F. Boquet, and G. Flandin. 2010. Robust $H_{\infty} / H_{-}$thruster failure detection and isolation with application to the LISA Pathfinder spacecraft. AIAA Guidance, Navigation and Control Conference. Toronto, Ontario: AIAA.

18. Wu, Q., and M. Saif. 2009. Model-based robust fault diagnosis for satellite control systems using learning and sliding mode approaches. J. Computers 4(10):1022-32.

19. Henry, D., and A. Zolghadri. 2005. Design and analysis of robust residual generators for systems under feedback control. Automatica 41:251-64. 
20. Scherer, C., P. Gahinet, and M. Chilali. 1997. Multiobjective output-feedback control via LMI optimization. IEEE Trans. Automatic Control 42(7):896-911.

21. Chilali, M., P. Gahinet, and P. Apkarian. 1999. Robust pole placement in LMI regions. IEEE Trans. Automatic Control 44(12):2257-70.

22. Wied, B. 1998. Space vehicle dynamics and control. Reston, VA: American Institute of Aeronautics and Astronautics.

23. CNES, 1998. Cours de technologie spatiale, Techniques et Technologies des véhicules spatiaux. Vols. 1-3. CEPADUES-ED.

24. Wisniewski, R. 2000. Lecture notes on modelling of a spacecraft. Aalborg Universitet, Aldeling for Proceskontrol.

25. Irvin, D. J. 2001. A study of linear vs. nonlinear control techniques for the reconfiguration of satellite formations. Ph.D. Thesis. Department of Aeronautics and Astronautics - Graduate School of Engineering and Management - Air Force Institute of Technology - Air University - Air Education and Training Command. 